\title{
Perspectives on the future of occupational epidemiology in Canada
}

\section{Perspectives sur l'avenir de l'épidémiologie du travail au Canada}

\author{
Paul J. Villeneuve ${ }^{1,2} \cdot$ Marie-Élise Parent ${ }^{3,4}$ \\ Published online: 12 October 2021 \\ (c) The Author(s) under exclusive license to The Canadian Public Health Association 2021
}

Occupational epidemiology has a rich history. Those of us trained in epidemiology before the turn of the twenty-first century were taught with the stories of Percivall Pott, and John Snow's use of occupational clues to help decipher the cause of the Broad Street Pump cholera outbreak. The teaching of the method of indirect standardization and the 'healthy worker' bias were drawn from case studies of occupational cancer. In those days, and with apologies to the Whitehall study (Marmot et al., 1991), occupational health research almost invariably focussed on cancer, and as pointed out by Loomis (Loomis et al., 2018), much of our understanding of the causes of cancer was first drawn from studies of workers. Historically, Canadian researchers made important contributions to this area, including studies that quantified cancer risks in miners (McDonald et al., 1971; Morrison et al., 1998), electric utility workers (Theriault et al., 1994), and farmers (Ritter et al., 1990). There is still a need to undertake occupational studies of cancer given that new substances continue to be introduced into workplaces, and there remain a large number of substances for which there are inadequate data to assess carcinogenicity.

Despite this need, the internationally respected occupational epidemiology research programs at McGill University and University of Toronto are a shadow of what

Paul J. Villeneuve

PaulVilleneuve@CUNET.CARLETON.CA

1 CHAIM Research Centre, Carleton University, Ottawa, ON, Canada

2 School of Mathematics and Statistics, Carleton University, Ottawa, ON, Canada

3 Unité d'épidémiologie et biostatistique, Centre Armand-Frappier Santé Biotechnologie, Institut national de la recherche scientifique, Laval, QC, Canada

4 École de santé publique, Université de Montréal, Montréal, QC, Canada they were 25 years ago. While newly introduced graduate programs in occupational health (for example at Ryerson University and Memorial University) hold promise, overall the capacity for occupational epidemiology in Canada has greatly diminished. While attaining funding remains difficult for all Canadian health researchers, support for occupational health research barely has a pulse. So much so that the Canadian Institutes of Health Research (CIHR) does not even track funding in this area. Occupational health researchers in Canada depend on a piecemeal network of provincial agencies that tend to support regional or localized studies.

The need for occupational health research, and training in this discipline, remains as critically important as ever. Mastromatteo provided an overview of priorities in occupational safety and health in this very journal in 1976 (Mastromatteo, 1976). He was prescient when identifying the need for future studies to consider mixtures of exposures when assessing occupational health risks. The recent SARSCOV-2 pandemic highlights the relevance of occupation as an important determinant of health. The pandemic has fundamentally changed the way we work. On one hand, it has opened opportunities for remote working; on the other, this has resulted in further reducing separation between home and work life. Some have posited that it has increased adverse health risks for some as, for many occupations, it has increased the number of working hours (Pega et al., 2021). The dichotomy between essential and non-essential workers is now apparent to most Canadians. As are the clear differences in equity in our workforce, with some workers, often of lower socio-economic status, facing barriers to screening, testing, and vaccination for COVID-19; these inequities surely apply to many other health conditions.

While Mastromatteo drew attention to the impact of 'women's liberation' on the workforce in 1976, and despite CIHR's call to arms to explore sex-/gender-related differences in risk, there have been relatively few occupational 
studies of women. Epidemiological literature suggests that, for more commonly studied exposures such as cigarette smoking and air pollution, women are more vulnerable than men. Sex or gender differences in risk remain a highly important research question area given that we often use risk estimates from occupational studies to quantify environmental risks of the same exposures occurring at much lower levels.

Opportunities in the field of occupational epidemiology abound. There have been substantial advances in exposure assessment and statistical methods, and increased ability to do quantitative risk assessments using biomarkers, cell phone apps, and omic technologies. The latter represent tools to discover biomarkers and provide opportunities for occupational health research with the capacity to study large sets of biological molecules (Vlaanderen et al., 2010). The concept of the exposome has gained traction where we combine exposure data from work and home environments to better understand health risks throughout the lifespan. There is a need for an improved understanding of how the work environment impacts a wide array of health outcomes, from injuries, cancer, and cardiovascular disease, to mental health. Importantly, there has been a transition with a gradual increase in 'white-collar' occupations, which to a greater extent can carry with them their own hazardous health conditions, including inactivity, stress, and ergonomics. Historically, two cycles of the UK Whitehall study (Marmot et al., 1991) have provided important insights on some of the cardiovascular behavioural risk factors, but more work is clearly needed. Relatedly, there is a need to recognize the opportunities for carrying out health promotion and intervention studies within workplace settings to mitigate some of these harms.

In Canada, many more recent and informal industries operate under the radar of existing regulations. Examples include drive-share programs. There will be a pressing need going forward to understand the health impacts of those at the front lines of fighting the climate emergency. This again is a stark reminder that occupational research should not be held back by borders, as Canada is part of a global economy. Perhaps more salient, however, is that we often rely on goods produced in other nations where workers do not have similar safeguards in place. Until recently, Canada was one of the world's largest exporters of asbestos, and despite the fact that these materials were banned from export in 2018, they will continue to claim lives abroad for decades. Canadians also rely on workers elsewhere for recycling of electronic waste. Occupational epidemiology in low-middle-income countries is seriously lacking. We have a public health responsibility to understand practices in these countries and take efforts to mitigate harms, particularly given that their work is hazardous and to the benefit of wealthy countries.
There are data gaps that remain glaring. Occupational data are not routinely collected in many of our administrative datasets. There is a delay of years before we can analyze national data from death registration. Cancer cases diagnosed in the province of Quebec from 2011 onward have yet to be submitted to the Canadian Cancer Registry (Brenner et al., 2020). As a result, while other jurisdictions are poised to carry out analyses to assess occupational risks of COVID-19 by occupation (Chen et al., 2021), we are forced to wait it out. Canada is uniquely positioned to undertake occupational health research given its capability in record linkage, but first, relevant data need to be captured such that they can be linked with comprehensive health outcome data.

As we anticipate a return to normalcy from the global pandemic, occupational epidemiology should be recognized as a priority for public health in Canada. This knowledge is essential to support primary prevention, surveillance, and compensation activities, as well as identify disease causes in the general population (Loomis et al., 2018). Going forward, our aim should be to expand and enhance the training and funding for these activities.

Paul Villeneuve, CJPH Senior Editor; Professor, Carleton University

Marie-Élise Parent, Professor, Institut national de la recherche scientifique

\section{Éditorial}

L'épidémiologie du travail a un riche passé. Ceux et celles d'entre nous qui avons étudié l'épidémiologie avant le début du $21^{\mathrm{e}}$ siècle ont appris les histoires de Percivall Pott et l'utilisation par John Snow d'indices professionnels pour décrypter la cause d'une éclosion de choléra, soit la célèbre pompe à eau de Broad Street. On nous a enseigné la méthode de la standardisation indirecte et «l'effet du travailleur bien portant » à partir d'études de cas de cancers professionnels. À l'époque, et avec toutes nos excuses à l'étude Whitehall (Marmot et al., 1991), les études sur la santé du travail portaient presque invariablement sur le cancer; comme l'a fait remarquer Loomis (Loomis et al., 2018), notre compréhension des causes du cancer provenait d'abord en majeure partie d'études sur des travailleurs. Par le passé, des chercheurs canadiens ont apporté d'importantes contributions au domaine, notamment avec des études chiffrant les risques de cancer chez les mineurs (McDonald et al., 1971; Morrison et al., 1998), les ouvriers des services publics d'électricité (Theriault et al., 1994) et les agriculteurs (Ritter et al., 1990). Il est encore nécessaire de mener des études sur l'exposition professionnelle aux cancérogènes, car on ne cesse d'introduire de nouvelles substances en milieu de travail, et il reste un grand nombre de substances dont il est impossible d'évaluer la cancérogénicité par manque de données. 
En dépit de ce besoin, les programmes de recherche en épidémiologie du travail de l'Université McGill et de l'Université de Toronto, respectés dans le monde entier, ne sont que l'ombre de ce qu'ils étaient il y a 25 ans. De nouveaux programmes de troisième cycle en santé du travail (par exemple à l'Université Ryerson et à l'Université Memorial) sont prometteurs, mais dans l'ensemble, la capacité en épidémiologie du travail au Canada a fortement diminué. Tous les chercheurs en santé canadiens ont du mal à obtenir du financement, mais le soutien à la recherche en santé du travail est sous respirateur artificiel. Les Instituts de recherche en santé du Canada (IRSC) ne suivent même pas l'évolution du financement dans ce domaine. Les chercheurs en santé du travail du Canada dépendent d'un réseau fragmentaire d'organismes provinciaux qui tendent à soutenir des études régionales ou localisées.

Le besoin de recherche en santé du travail, et de formation dans cette discipline, est aussi criant que jamais. Mastromatteo avait brossé un portrait des priorités en sécurité et santé du travail dans la présente revue en 1976 (Mastromatteo, 1976). Il était en avance sur son époque lorsqu'il a défini le besoin, dans les études futures, de tenir compte des expositions mixtes pour évaluer les risques d'exposition professionnelle. La récente pandémie de SRAS-CoV-2 met en évidence l'importance de la profession en tant que déterminant de la santé. La pandémie a fondamentalement changé la façon dont nous travaillons. D'un côté, elle a ouvert la possibilité de travailler à distance; de l'autre, elle a encore réduit la séparation entre le travail et la vie personnelle. Il a été avancé qu'elle a accru les risques pour la santé chez certains travailleurs en faisant augmenter, dans plusieurs professions, le nombre d'heures de travail (Pega et al., 2021). La dichotomie entre les travailleurs essentiels et non essentiels est maintenant apparente pour la plupart des Canadiens, tout comme les inégalités claires au sein de la main-d'oeuvre, où certains travailleurs, souvent de faible statut socioéconomique, font face à des obstacles au dépistage, aux tests et à la vaccination contre la COVID-19. Ces iniquités valent sûrement aussi pour bien d'autres maladies.

Mastromatteo attirait l'attention sur l'impact de la « libération de la femme » sur la main-d'œuvre en 1976, mais malgré l'appel aux armes des IRSC pour que les différences de risque liées au sexe ou au genre soient explorées, il y a eu relativement peu d'études sur l'exposition professionnelle des femmes. La littérature épidémiologique donne à penser que pour les expositions les plus communément étudiées, comme l'usage de la cigarette et la pollution atmosphérique, les femmes sont plus vulnérables que les hommes. Les différences de risque liées au sexe ou au genre demeurent un domaine de recherche très important, car nous faisons souvent appel aux estimations du risque tirées d'études sur l'exposition professionnelle pour chiffrer les risques environnementaux des mêmes expositions survenant à des niveaux beaucoup plus faibles.
Les possibilités foisonnent dans le domaine de l'épidémiologie du travail. Il y a eu d'importantes avancées dans l'évaluation des expositions et les méthodes statistiques, et nous avons amélioré notre capacité d'effectuer des appréciations quantitatives du risque à l'aide de biomarqueurs, d'applications pour téléphones cellulaire et des technologies omiques. Ces dernières sont des outils de découverte de biomarqueurs qui ouvrent des possibilités de recherche en santé du travail, car elles permettent d'étudier de vastes ensembles de molécules biologiques (Vlaanderen et al., 2010). Le concept de l'exposome, qui combine les données sur les expositions dans les milieux de vie et de travail pour mieux comprendre les risques pour la santé de la conception à la mort, gagne du terrain. Il est nécessaire de mieux comprendre les incidences du milieu de travail sur un vaste éventail de résultats cliniques - des blessures, cancers et maladies cardiovasculaires à la santé mentale. Surtout, il y a eu une augmentation progressive des professions de « cols blancs », qui peuvent dans une plus grande mesure être assorties de leurs propres troubles de santé, comme la sédentarité, le stress et les problèmes ergonomiques. Par le passé, deux des cycles de l'étude Whitehall du RoyaumeUni (Marmot et al., 1991) ont apporté d'importants éclaircissements sur quelques-uns des facteurs de risques comportementaux des maladies cardiovasculaires, mais il est clair qu'il faudrait pousser la recherche à ce sujet. Corollairement, il faut reconnaître les possibilités d'effectuer des études de promotion de la santé et d'intervention en milieu de travail pour atténuer certains de ces méfaits.

$\mathrm{Au}$ Canada, beaucoup d'autres industries récentes et informelles échappent à la réglementation existante. Les programmes d'autopartage en sont un exemple. Il existe maintenant un besoin pressant de comprendre leurs effets sanitaires au front de la bataille contre l'urgence climatique. C'est un autre rappel brutal que la recherche sur les expositions professionnelles ne doit pas être empêchée par les frontières, car le Canada fait partie de l'économie mondiale. Plus important encore peut-être, nous dépendons souvent de biens produits dans d'autres pays où les travailleurs n'ont pas les mêmes protections. Jusqu'à tout récemment, le Canada était l'un des plus grands exportateurs mondiaux d'amiante, et malgré l'interdiction d'exporter les produits de l'amiante depuis 2018, ceux-ci continueront de coûter des vies à l'étranger pendant des dizaines d'années. Les Canadiens dépendent aussi des travailleurs étrangers pour le recyclage des déchets électroniques. L'épidémiologie du travail dans les pays à faible revenu et à revenu intermédiaire fait cruellement défaut. La santé publique a l'obligation de connaitre les pratiques dans ces pays et de faire des efforts pour en atténuer les méfaits. À plus forte raison parce que c'est un travail dangereux qui profite aux pays riches.

Il reste des lacunes statistiques flagrantes à combler. Les données sur les expositions professionnelles ne sont pas systématiquement collectées dans un grand nombre de 
nos jeux de données administratives. Il y a un décalage de plusieurs années entre la production et l'analyse des données nationales d'enregistrement des décès. Les cas de cancer diagnostiqués au Québec depuis 2011 n'ont pas encore été soumis au Registre canadien du cancer (Brenner et al., 2020). Résultat : alors que d'autres sphères de compétence s'apprêtent à effectuer des analyses pour évaluer les risques d'exposition à la COVID-19 par profession (Chen et al., 2021), nous sommes obligés d'attendre. Le Canada est particulièrement bien placé pour faire de la recherche en santé du travail, vu ses capacités de couplage de dossiers, mais d'abord, les données pertinentes doivent être saisies de manière à pouvoir être maillées aux données globales sur les résultats cliniques.

Avec le retour à la normale attendu après la pandémie mondiale, il faudrait que l'épidémiologie du travail soit reconnue comme une priorité pour la santé publique au Canada. Ce savoir scientifique est essentiel pour appuyer les activités de prévention primaire, de surveillance et d'indemnisation et pour déterminer les causes de maladie dans la population générale (Loomis et al., 2018). Désormais, notre objectif devrait être d'élargir et d'améliorer le financement de ces activités et la formation à leur sujet.

Paul Villeneuve, Rédacteur scientifique adjoint, RCSP; professeur, Université Carleton

Marie-Élise Parent, Professeure, Institut national de la recherche scientifique

\section{References/Références}

Brenner, D. R., Weir, H. K., Demers, A. A., Ellison, L. F., Louzado, C., Shaw, A., et al. (2020). Projected estimates of cancer in Canada in 2020. CMAJ, 192(9), E199-E205. https://doi.org/10.1503/cmaj. 191292

Chen, Y. H., Glymour, M., Riley, A., Balmes, J., Duchowny, K., Harrison, R., et al. (2021). Excess mortality associated with the COVID-19 pandemic among Californians 18-65 years of age, by occupational sector and occupation: March through November 2020. PLoS One, 16(6), e0252454. https://doi.org/10.1371/journal.pone.0252454

Loomis, D., Guha, N., Hall, A. L., \& Straif, K. (2018). Identifying occupational carcinogens: An update from the IARC Monographs. Occup Environ Med, 75(8), 593-603. https://doi.org/10.1136/oemed-2017-104944

Marmot, M. G., Smith, G. D., Stansfeld, S., Patel, C., North, F., Head, J., et al. (1991). Health inequalities among British civil servants: The Whitehall II study. Lancet, 337(8754), 1387-1393. https:// doi.org/10.1016/0140-6736(91)93068-k

Mastromatteo, E. (1976). Keynote address 1: Occupational health--the international scope: Lessons for Canada. Can J Public Health, 67 suppl 2, 9-15. Retrieved from https://www.ncbi.nlm.nih.gov/ pubmed/991059

McDonald, J. C., McDonald, A. D., Gibbs, G. W., Siemiatycki, J., \& Rossiter, C. E. (1971). Mortality in the chrysotile asbestos mines and mills of Quebec. Arch Environ Health, 22(6), 677-686. https://doi.org/10.1080/00039896.1971.10665923

Morrison, H. I., Villeneuve, P. J., Lubin, J. H., \& Schaubel, D. E. (1998). Radon-progeny exposure and lung cancer risk in a cohort of Newfoundland fluorspar miners. Radiat Res, 150(1), 58-65. Retrieved from https://www.ncbi.nlm.nih.gov/pubmed/9650603

Pega, F., Nafradi, B., Momen, N. C., Ujita, Y., Streicher, K. N., Pruss-Ustun, A. M., et al. (2021). Global, regional, and national burdens of ischemic heart disease and stroke attributable to exposure to long working hours for 194 countries, 2000-2016: A systematic analysis from the WHO/ILO Joint Estimates of the Work-related Burden of Disease and Injury. Environ Int, 154, 106595. https://doi.org/10.1016/j.envint.2021.106595

Ritter, L., Wigle, D. T., Semenciw, R. M., Wilkins, K., Riedel, D., \& Mao, Y. (1990). Mortality study of Canadian male farm operators: Cancer mortality and agricultural practices in Saskatchewan. Med Lav, 81(6), 499-505. Retrieved from https://www.ncbi.nlm.nih. gov/pubmed/2100765

Theriault, G., Goldberg, M., Miller, A. B., Armstrong, B., Guenel, P., Deadman, J., . . . et al. (1994). Cancer risks associated with occupational exposure to magnetic fields among electric utility workers in Ontario and Quebec, Canada, and France: 1970-1989. Am J Epidemiol, 139(6), 550-572. doi:https://doi.org/10.1093/ oxfordjournals.aje.a117046

Vlaanderen, J., Moore, L. E., Smith, M. T., Lan, Q., Zhang, L., Skibola, C. F., et al. (2010). Application of OMICS technologies in occupational and environmental health research; current status and projections. Occup Environ Med, 67(2), 136-143. https://doi. org/10.1136/oem.2008.042788

Publisher's note Springer Nature remains neutral with regard to jurisdictional claims in published maps and institutional affiliations. 